\title{
Computational Nonlinear Dynamics Model of Percept Switching with Ambiguous Stimuli
}

\author{
Norbert Fürstenau \\ German Aerospace Center, Institute of Flight Guidance, Lilienthalplatz 7 \\ D-38108 Braunschweig, Germany \\ norbert.fuerstenau@dlr.de
}

Simulation results of bistable perception due to ambiguous visual stimuli are presented which are obtained with a behavioral nonlinear dynamics model using perception-attention-memory coupling. As a kind of minimum architecture representing the Thalamo-Cortical V4InferoTemporal-PraeFrontal-V4 ("what") loop the basic model couples the dynamics of a macroscopic perception state order parameter with an adaptive attention (feedback gain) control parameter with reentrant delay $\mathrm{T}$ and additive band limited attention noise (Fürstenau 2006, 2007). Quasiperiodic perceptual switching is induced by attention fatigue with a perception bias which balances the relative duration of the alternative percepts, corresponding to the well known Synergetics model of Ditzinger and Haken (1989). Mean perceptual duration times of $2-5 \mathrm{~s}$ of the Gamma-distributed dwell time statistics are predicted in agreement with experimental results reported in the literature, if a feedback delay $\mathrm{T}$ of $40 \mathrm{~ms}$ is assumed which is typical for cortical reentrant loops and the stimulus-V1 latency (Lamme 2003). Numerically determined perceptual transition times of $3-5 \mathrm{~T}$ are in reasonable agreement with stimulus-conscious perception delay of 150 - 200 ms. Periodic stimulus simulations as a function of stimulus off-time yields the reversal rate variation in surprisingly good quantitative agreement with classical experimental results of Orbach et.al.(1966) when selecting adaptation and recovery time constants of $1-2 \mathrm{~s}$. As an additional feature memory effects are introduced by allowing for the slow adaptation of the perception bias parameter via coupling to the perception state. They are quantified by calculation of the self similarity (Hurst) parameter $\mathrm{H}$ of the reversal time series $(\mathrm{H}>0.5)$. The simulations exhibit long range correlations, i.e. the fractal character of the perceptual duration times in agreement with recent experimental results of Gao et al. (2006). This finding again fits into the proposed picture of underlying nonlinear brain dynamics as derived from analysis and theoretical modeling of EEG time series (e.g. (Lutzenberger et.al. 1995)). Also a straightforward extension appears possible for modeling top-down control of eye movements.

Ditzinger, T., Haken, H.(1989). Oscillations in the Perception of Ambiguous Patterns. Biol. Cybern. 61, 279-287

Gao, J.B., Billock, V.A., Merk, I., Tung, W.W., White, K.D., Harris, J.G., Roychowdhury, V.P. (2006). Inertia and Memory in Ambiguous Visual Perception, Cogn. Process vol. 7, 105-112

Fürstenau, N. (2006). Modelling and Simulation of spontaneous perception switching with ambiguous visual stimuli in augmented vision systems. Lecture Notes in Artificial Intelligence 4021, Springer, Berlin, New York, 20 -31

Fürstenau, N. (2007). A Computational Model of Bistable Perception-Attention Dynamics with Long Range Correlations. Lecture Notes in Artificial Intelligence 4667, Springer, Berlin, New York, 251 263

Ito, J., Nikolaev, A.R., Luman, M., Aukes, M.F., Nakatani, C., van Leeuwen, C. (2003). Perceptual switching, eye movements, and the bus paradox. Perception 32, 681 - 698

Lamme, V.A.F. (2003). Why visual attention and awareness are different. Trends in cognitive Sciences 7 12-18

Orbach. J., Zucker, E., Olson, R. (1966). Reversibility of the Necker Cube: VII. Reversal rate as a function of figure-on and figure-off durations. Percept. and Motor Skills, 22, 615-618

Lutzenberger, W., Preissl, H., Pulvermüller, F. (1995): Fractal dimension of electroencephalographic time series and underlying brain processes. Biol. Cybern. 73 477-482 